\title{
Very Metal-poor Stars Observed by the RAVE Survey
}

\author{
Gal Matijevič ${ }^{1}$ and the RAVE Collaboration \\ ${ }^{1}$ Leibniz-Institut für Astrophysik Potsdam (AIP), \\ An der Sternwarte 16, 14482 Potsdam, Germany \\ email: gmatijevic@aip.de
}

\begin{abstract}
Radial Velocity Experiment (RAVE) observed 500,000 southern sky stars between 2003 and 2013 in the infra-red calcium triplet (CaII) spectral region. In this study we extended the analysis of RAVE very metal-poor stars $([\mathrm{Fe} / \mathrm{H}]<-2)$ presented by Fulbright et al. $(2010)$. We employed a novel method for identifying the metal-poor stars and developed a tool for modeling CaII lines where we also modeled the background noise to avoid systematical biases in the equivalent width $(\mathrm{EW})$ measurements. Final metallicity values were derived with a flexible calibration approach using only 2MASS photometric data and EW measurements obtained from the RAVE spectra.
\end{abstract}

Keywords. Metal-poor stars, RAVE survey, Gaussian processes

\section{Introduction}

Metal-poor stars have been extensively used for stellar and galactic archeology studies over the last few decades (Beers \& Christlieb 2005). They offer an insight into the nucleosynthesis in the early Galaxy and subsequent metal enrichment by the supernovae, and allow us to study the conditions in the young Universe in which they were born. Large-scale all-sky spectroscopic surveys like RAVE offer a great opportunity to discover new very-metal poor stars and measure their metallicities.

\section{Metal-poor spectra identification}

RAVE's 4th data release (Kordopatis et al. 2013, DR4) includes atmospheric parameters including metallicities for most of the observed stars. However, due to the lack of significant features in the spectra of very metal-poor stars, the parameters become less reliable with decreasing metallicity and the spectra need to be reanalyzed. The first step is to correctly identify all metal-poor candidates. We used a method called t-SNE (van der Maaten 2014) to compute a 2D projection of all spectra in the database (similarly as in Matijevič et al. 2012). The method groups morphologically similar spectra together so it is easy to identify and isolate the group of $\sim 3000$ metal-poor FGK-type candidates for which we computed better metallicity estimates (Fig. 1).

\section{Line profile modeling}

Deriving abundances requires measuring the equivalent widths. Usually this is done by defining a line profile and fitting it to the spectral lines by minimizing the $\chi^{2}$ statistic. The EW is measured by integrating the profile. Problems arise if the conditions under which the $\chi^{2}$ is a good estimator are violated, i.e. when noise is not independent and 

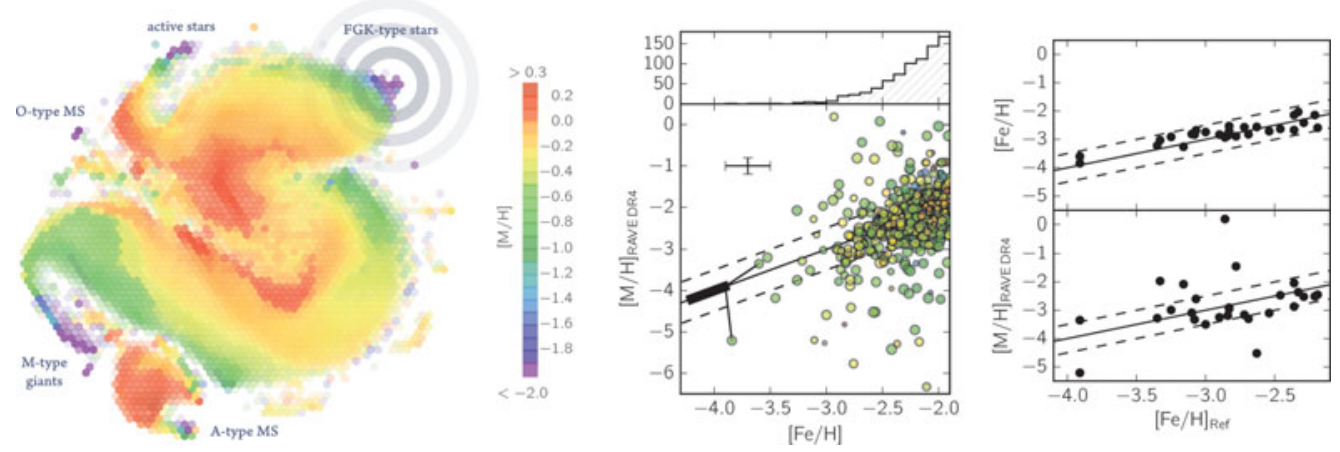

Figure 1. Left: t-SNE projection of RAVE spectra with $S / N>10$. Circled region is occupied by the spectra that were reanalyzed. Center: Comparison between the new metallicities and those provided by DR4. Right: Comparison between new, DR4, and high resolution reference $[\mathrm{Fe} / \mathrm{H}]$ values of a subset of stars.

identically distributed. This often happen because a) the profile of the targeted line also includes weaker lines, and b) reduction and normalization introduce variations or offsets in the continuum. Ignoring these problems can lead to biased EW measurements and underestimated uncertainties because the solution is too stiff. We corrected for these difficulties by introducing additional terms in the likelihood function to model the correlated noise using Gaussian processes (Rasmussen \& Williams 2006). This way we computed less precise but more accurate EWs.

\section{Metallicity calibration}

Equivalent widths of CaII triplet lines correlate very well with metallicity, but also depend strongly on the luminosity which introduces degeneracy: spectra of less metalpoor dwarfs resemble spectra of more metal-poor giants. For clusters this can be resolved by adding a term to the calibration relation that describes the distance to the cluster's horizontal branch. Unfortunately, this method cannot be used for field stars at unknown distances. Instead, we relied on measured EWs and 2MASS $J H K_{S}$ colors. Motivated by The Cannon (Ness et al. 2015), we defined the calibration relation by training the model on a subset for which we have high-resolution data form Ruchti et al. (2013) and Carrera et al. (2013). Afterwards, we evaluated the measured EWs and colors of the remaining stars in the metal-poor sample to obtain their metallicities. This was done with Gaussian processes to give the model enough flexibility. We were able to identify several hundred new very-metal poor stars. Comparison with the metallicities measured form the high resolution spectra confirms that new values are indeed much more accurate (Fig. 1).

\section{References}

Beers, T. C. \& Christlieb, N. 2005, ARA\&A, 43, 531

Carrera, R., Pancino, E., Gallart, C., et al. 2013, MNRAS, 434, 1681

Fulbright, J. P., Wyse, R. F. G., \& Ruchti, G. R., et al. 2010, ApJ, 724, L104

Kordopatis, G., Gilmore, G., Steinmetz, M., et al. 2013, AJ, 146, 134

Matijevič, G., Zwitter, T., Bienaym, O., et al. 2012, ApJS, 200, 14

Ness, M., Hogg, D. W., \& Rix, H. -W., et al. 2015, ApJ, 808, 16

Rasmussen, C. E. \& Williams, C. 2006, MIT Press

Ruchti, G. R., Bergemann, M., Serenelli, A., et al. 2013, MNRAS, 429, 126

van der Maaten, L. J. P.. 2014, Journal of Machine Learning Research, 15, 3221 ? 